\title{
USO NÃO PRESCRITO DE CLORIDRATO DE METILFENIDATO ENTRE ESTUDANTES UNIVERSIRÁRIOS ${ }^{1}$
}

\author{
Jefferson Marlon de Medeiros Pereira Maciel, Universidade Federal de Campina Grande \\ (UFCG), jeffersonmmpmaciel@hotmail.com \\ Andreza Guedes Barbosa Ramos, Universidade Federal de Campina Grande (UFCG), \\ andrezaurca@gmail.com
}

\begin{abstract}
RESUMO
Na contemporaneidade, estudantes universitários saudáveis têm usado substâncias psicoativas com o objetivo de potencializar as atividades mentais, ao permitir que eles estudem por mais horas e otimizem sua eficiência acadêmica. O cloridrato de metilfenidato é um dos estimulantes mais prescritos no mundo e controlado para o tratamento farmacológico do transtorno de déficit de atenção e hiperatividade (TDAH), mas tornou-se proeminente no campo popular pelo seu uso como Aprimoramento Cognitivo Farmacológico. O estudo trata-se de uma revisão integrativa da literatura que busca responder a seguinte pergunta norteadora: Quais as contribuições e possíveis efeitos adversos que o Cloridrato de Metilfenidato promovem nos jovens e adultos que o utilizam indiscriminadamente na busca por um estado de alerta necessário à rotina acadêmica?. Foram encontradas 318 publicações nas bases de dados eletrônicos MedLINE, LILACS e SciELO, das quais após sistematização para o escopo da revisão, restaram 06 publicações que são detalhadas nesse estudo. Há um número insuficiente de estudos que tratam sobre o uso de psicoestimulantes entre estudantes saudáveis na literatura brasileira. Assim, é preciso avaliar os impactos do uso de estimulantes na prática clínica, a fim de minimizar possíveis efeitos adversos, avaliar a dose considerada segura e coibir o uso de determinadas substâncias.
\end{abstract}

PALAVRAS-CHAVE: metilfenidato; estudantes; uso de medicamentos.

\section{NOT PRESCRIBED USE OF METHYLPHENIDATE CHLORIDRATE BETWEEN UNIVERSITY STUDENTS}

\begin{abstract}
In today's world, healthy college students have used psychoactive substances to potentiate mental activity by allowing them to study for longer hours and optimize their academic effectiveness. Methylphenidate hydrochloride is one of the most prescribed stimulants in the world and controlled for the pharmacological treatment of Attention Deficit Hyperactivity Disorder (ADHD), but it has become prominent in the popular field for its use as Cognitive Pharmacological Enhancement. The study is an integrative review of the literature that seeks to answer the following guiding question: What are the contributions and possible adverse effects that Methylphenidate Hydrochloride promotes

${ }^{1} \mathrm{O}$ presente trabalho não contou com apoio financeiro de nenhuma natureza para sua realização.
\end{abstract}


in young adults and adults who use it indiscriminately in the search for a necessary alertness routine Academic?. A total of 318 publications were found in the electronic databases MedLINE, LILACS and SciELO, of which after systematization for the scope of the review, there were 06 publications that are detailed in this study. There is an insufficient number of studies dealing with the use of psychostimulants among healthy students in Brazilian literature. Thus, it is necessary to evaluate the impacts of the use of stimulants in clinical practice, in order to minimize possible adverse effects, to evaluate the dose considered safe and to restrain the use of certain substances.

KEYWORDS: methylphenidate; students; use of medicines.

\title{
USO NO PRESCRITO DE CLORIDRATO DE METILFENIDATO ENTRE ESTUDIANTES UNIVERSITARIOS
}

\begin{abstract}
RESUMEN
En la contemporaneidad, estudiantes universitarios sanos han usado sustancias psicoactivas con el objetivo de potenciar las actividades mentales, al permitir que ellos estudien por más horas y optimizar su eficiencia académica. El clorhidrato de metilfenidato es uno de los estimulantes más prescritos en el mundo y controlado para el tratamiento farmacológico del trastorno de déficit de atención e hiperactividad (TDAH), pero se ha vuelto prominente en el campo popular por su uso como Mejoramiento Cognitivo Farmacológico. El estudio se trata de una revisión integrativa de la literatura que busca responder a la siguiente pregunta orientadora: ¿Cuáles son las contribuciones y posibles efectos adversos que el Clorhidrato de Metilfenidato promueven en los jóvenes y adultos que lo utilizan indiscriminadamente en la búsqueda de un estado de alerta necesario a la rutina ". Se encontraron 318 publicaciones en las bases de datos electrónicos MedLINE, LILACS y SciELO, de las cuales después de sistematización para el alcance de la revisión, quedaron 06 publicaciones que se detallan en ese estudio. Hay un número insuficiente de estudios que tratan sobre el uso de psicoestimulantes entre estudiantes sanos en la literatura brasileña. Así, es necesario evaluar los impactos del uso de estimulantes en la práctica clínica, a fin de minimizar posibles efectos adversos, evaluar la dosis considerada segura y cohibir el uso de determinadas sustancias.
\end{abstract}

PALABRAS CLAVES: metilfenidato; estudiantes; uso de medicamentos.

\section{INTRODUÇÃO}

$\mathrm{Na}$ contemporaneidade, algumas substâncias psicotrópicas ao modificarem os modos de ser, de viver e de trabalhar, apenas secundariamente são utilizadas para minimização do sofrimento e como estratégia para potencializar performances escolares e laborais. Elevados níveis de produtividade em curto prazo, com baixo custo e alta qualidade, são imperativos colocados pela ditadura do sucesso a qualquer custo (BRANT; CARVALHO, 2012, p. 624).

A universidade é um ambiente que incentiva a aprendizagem e a pesquisa para desenvolver ciência, e isso requer um alto nível de trabalho e dedicação pelos estudantes, 
tornando-a um espaço altamente competitivo. Nestas circunstâncias, estudantes universitários saudáveis têm usando substâncias psicoativas com o objetivo de potencializar as atividades mentais, ao permitir que eles estudem por mais horas e otimizem sua eficiência acadêmica (FINGER; SILVA; FALAVIGNA, 2013, p. 286).

Medicamentos psicoestimulantes são aqueles com capacidade de aumentar o estado de alerta e a motivação, diminuindo a necessidade de descanso, além de possuírem propriedades antidepressivas, de melhora no humor e no desempenho cognitivo. As principais substâncias utilizadas para essa finalidade são: cafeína, metilfenidato, modafinil, piracetam, bebidas energéticas e anfetaminas (MORGAN et al., 2017, p. 103).

O cloridrato de metilfenidato $\left(\mathrm{MPH}\right.$; Concerta ${ }^{\circledR}$, Ritalina $\left.{ }^{\circledR}\right)$ é um dos estimulantes mais prescritos no mundo e controlado em muitos países para o tratamento farmacológico do transtorno de déficit de atenção e hiperatividade (TDAH) e casos específicos de transtorno depressivo e fadiga. Quando indicada corretamente, em doses adequadas, as chances da droga levar ao vício são muito baixas, sendo considerada assim uma medicação segura com nível satisfatório de efeitos colaterais. Seu mecanismo de ação é de estimulo do sistema nervoso central (SNC) ao bloquear a receptação da dopamina, elevando a sua concentração e de norepinefrina no espaço sináptico (CESAR et al., 2012, p. 183).

Apesar da restrição citada, são conhecidos três usos não clínico desse fármaco o recreativo, para aumentar o tempo de vigília e disposição durante o lazer; o estético, para auxiliar o emagrecimento; e o aprimoramento cognitivo, para melhorar o desempenho cognitivo profissional e acadêmico (BARROS; ORTEGA, 2011, p.352).

O uso de MPH para aprimoramento cognitivo tornou-se proeminente no campo científico e popular principalmente após um artigo de 2008 publicado on-line no Jornal Nature intitulado "Resultados da pesquisa: veja quem é Doping". A novidade apresentada no artigo está na possibilidade de usar remédios e outras intervenções biotecnológicas para modificar o processo cerebral com o objetivo de aprimorar memória, humor e atenção em pessoas saudáveis (BARROS; ORTEGA, 2011, p.352). No entanto, esse uso é controverso, uma vez que não há ainda evidências de sua eficácia nem do equilíbrio benefício-risco, além de haver potencial para abuso quando usadas em dose inadequadas e associação com uso de outras substâncias, como álcool e cocaína (SILVEIRA et al., 2014, p. 103). 
De acordo com os registros do United States Department of Justice Drug Enforcement Administration (DEA), a produção de MPH aumentou 298\% no período de 1996 a 2006, o que tem contribuído para fomentar o debate sobre a adequação médica na identificação de casos de TDAH e uso indiscriminado por indivíduos que buscam nesse fármaco seus efeitos estimulantes, para fins hedonistas e na expectativa de aprimoramento cognitivo (CESAR et al., 2012, p. 183).

Evidencia-se que grande parte da prescrição e o consumo não terapêutico do metilfenidato, na contemporaneidade, o transformam em um autêntico gadget, ao serem vendidos com a promessa de modularem desejos, elevarem a autoestima e proporcionarem a sensação de ganho de energia (BRANT; CARVALHO, 2012, p. 630).

Assim, apesar de haver poucos estudos concluídos sobre o uso não médico do MPH no Brasil, a análise dos dados da presente investigação sugere que o Aprimoramento Cognitivo Farmacológico é um assunto atual e relevante, não apenas por esta prática estar relacionada à construção e manutenção da subjetividade dos indivíduos em uma sociedade que prioriza a melhoria da performance cognitiva, mas também pelo risco de esta interferir em questões de igualdade e justiça social (BARROS; ORTEGA, 2011, p.360). Sem contar, o fato de que o uso abusivo de drogas, além da dependência, ultrapassa o nível da clínica individual e configura-se como importante problema de saúde pública.

Nesse sentido, o objetivo do estudo é avaliar a prevalência do uso de metilfenidato entre estudantes universitários, fazendo distinção entre aqueles que o utilizam para tratamento de distúrbios neuropsiquiátricos e os usuários não clínicos, apresentando frequência e motivação para o uso, bem como os efeitos deletérios para a saúde.

\section{MÉTODOS}

Este estudo de abordagem qualitativa revisou a literatura para identificar os efeitos da automedicação e uso indiscriminado de Cloridrato de Metilfenidato (Ritalina®) entre estudantes universitários. Optou-se pela realização de uma revisão integrativa por ser a mais ampla abordagem metodológica referente às revisões, ao resumir a literatura empírica ou teórica para fornecer uma compreensão abrangente de um fenômeno particular (BROOME, 2016).

Para a produção dessa revisão foi usada a estrutura descrita por Botelho, Cunha, e Macedo (2011, p. 127), compreendida em seis etapas: identificação do tema e seleção da 
questão norteadora; estabelecimento dos critérios de inclusão e exclusão; identificação dos estudos selecionados; categorização dos estudos; análise e interpretação dos resultados; apresentação da síntese do conhecimento.

Dessa forma, o tema explorado nesta revisão integrativa foi a atuação do Cloridrato de Metilfenidato no cotidiano dos estudantes universitários, sendo definida a seguinte pergunta norteadora: Quais as contribuições e possíveis efeitos adversos que o Cloridrato de Metilfenidato promovem nos jovens e adultos que o utilizam indiscriminadamente na busca por um estado de alerta necessário à rotina acadêmica?

Para o levantamento dos artigos na literatura, realizou-se uma busca durante os meses de julho e agosto de 2017 nas seguintes bases de dados: Literatura Latino-Americana e do Caribe em Ciências da Saúde (LILACS), Medical Literature Analysis and Retrieval System Online (MedLINE) e Scientific Electronic Library Online (SciELO). Foram utilizados os seguintes descritores controlados combinados com operadores booleanos: "Estudantes", "Metilfenidato", "Efeitos de Drogas", e "Uso de Medicamentos".

Os critérios de inclusão definidos para a seleção dos artigos foram: artigos publicados nas bases de dados pesquisadas; artigos publicados em português ou inglês; trabalhos desenvolvidos no período de 2007 a 2016; disponibilidade on-line dos textos completos. Tomou-se o cuidado em excluir estudos sem elementos relevantes ao escopo da revisão e os artigos que apresentam duplicidade nas bases de dados.

\section{RESULTADOS}

Obteve-se o resultado de 52 publicações na base de dados SciELO, 84 na LILACS e 182 na MedLINE, totalizando 318 artigos que poderiam ter relação com o tema da pesquisa. Após leitura dos títulos, desse total, 259 não possuíam relação direta com o tema do estudo ou estavam indexados em mais de uma base de dados, restando 59 publicações que seguiram para a sistematização dos dados, como apresentado na Figura 1.

Após a coleta de dados, por uma análise descritiva, com o intuito de reunir o conhecimento produzido sobre o tema explorado na revisão, a amostra final desta foi definida por 06 artigos científicos, selecionados pelos critérios de inclusão previamente estabelecidos. Destes, 02 foram encontrados na base de dados SciELO, 03 na LILACS e 01 na MedLINE. A relação dos artigos e suas variáveis são apresentados no Quadro 1. 
Figura 1. Fluxograma do processo de seleção das publicações, Paraíba, Brasil, 2017.

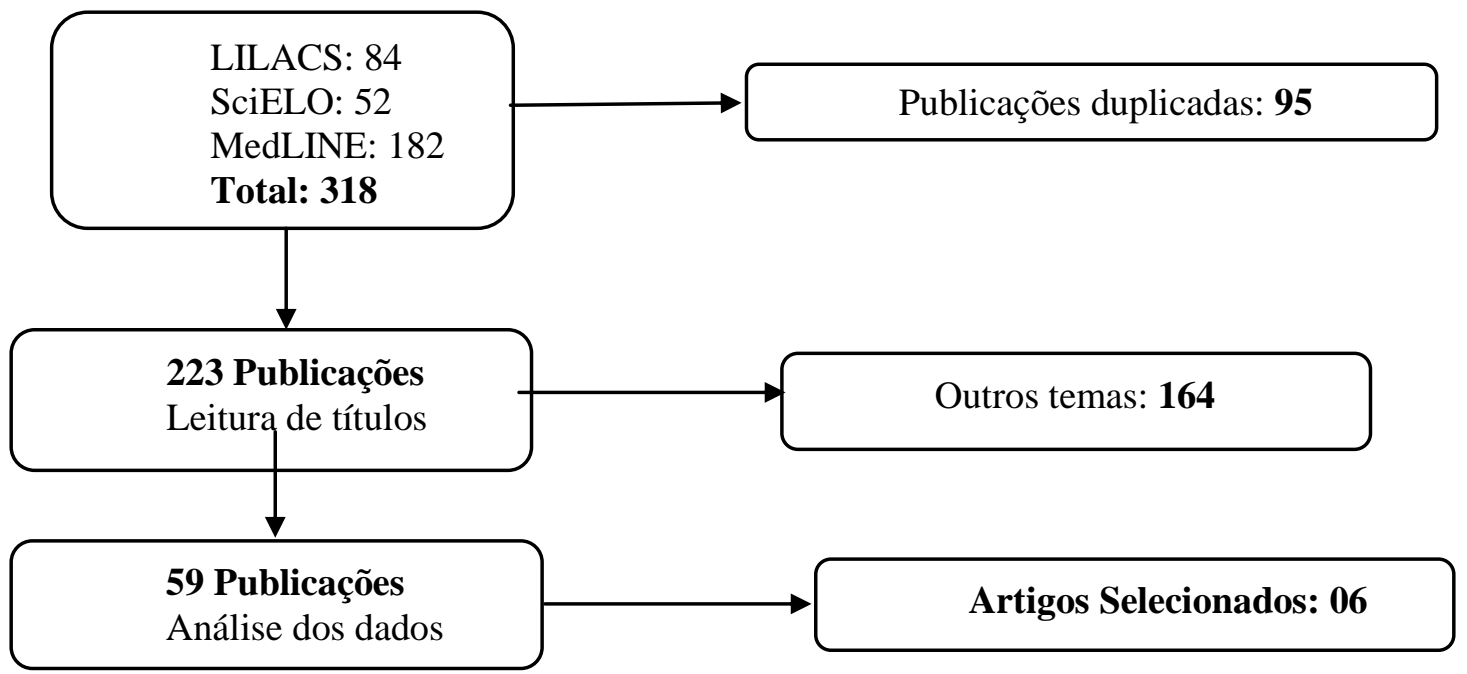


Quadro 1. Relação dos artigos incluídos no estudo e suas variáveis, Paraíba, Brasil, 2017.

\begin{tabular}{|c|c|c|c|}
\hline $\begin{array}{c}\text { Base de } \\
\text { Dados }\end{array}$ & Títulos & Autores & Objetivos \\
\hline \multirow{3}{*}{ LILACS } & $\begin{array}{l}\text { Uso prescrito de cloridrato de } \\
\text { metilfenidato e correlatos entre } \\
\text { estudantes } \\
\text { brasileiros }\end{array}$ & $\begin{array}{l}\text { CESAR, Eduardo Luiz Da } \\
\text { Rocha et al }\end{array}$ & $\begin{array}{l}\text { Estimar a prevalência do uso prescrito de } \\
\text { MPH e correlatos em uma amostra } \\
\text { populacional de universitários brasileiros }\end{array}$ \\
\hline & $\begin{array}{l}\text { Metilfenidato: } \quad \text { medicamento } \\
\text { gadget da contemporaneidade }\end{array}$ & $\begin{array}{lr}\text { BRANT, Luiz } & \text { Carlos; } \\
\text { CARVALHO, } & \text { Tales } \\
\text { Renato Ferreira } & \\
\end{array}$ & $\begin{array}{l}\text { Apresentar os principais aspectos do uso } \\
\text { não médico do metilfenidato }\end{array}$ \\
\hline & $\begin{array}{l}\text { Patterns of non-medical use of } \\
\text { methylphenidate among 5th and } \\
6 \text { th year students in a medical } \\
\text { school in southern Brazil }\end{array}$ & $\begin{array}{l}\text { SILVEIRA, Rodrigo da } \\
\text { Rosa } \text { et al }\end{array}$ & $\begin{array}{l}\text { Avaliar a prevalência do uso do } \\
\text { metilfenidato entre estudantes do } 5^{\circ} \text { e do } \\
6^{\circ} \text { ano de uma faculdade de medicina, } \\
\text { discriminar o uso com ou sem indicação } \\
\text { médica e correlacionar o uso de } \\
\text { metilfenidato com a ingestão de álcool }\end{array}$ \\
\hline MedLINE & $\begin{array}{l}\text { Use of methylphenidate among } \\
\text { medical students: a systematic } \\
\text { review }\end{array}$ & $\begin{array}{lr}\text { FINGER, } & \text { Guilherme; } \\
\text { SILVA, } & \text { Emerson } \\
\text { Rodrigues } & \text { da; } \\
\text { FALAVIGNA, } & \text { Asdrubal }\end{array}$ & $\begin{array}{l}\text { Avaliar os efeitos do metilfenidato no } \\
\text { aprimoramento cognitivo, na memória e } \\
\text { no desempenho em estudantes de } \\
\text { medicina }\end{array}$ \\
\hline \multirow[t]{2}{*}{ SciELO } & $\begin{array}{l}\text { Consumo de } \\
\text { Cerebrais por Estimulantes } \\
\text { Medicina de uma Universidade do } \\
\text { Extremo Sul do } \quad \text { Brasil: } \\
\text { Prevalência, Motivação e Efeitos } \\
\text { Percebidos }\end{array}$ & $\begin{array}{l}\text { MORGAN, Henri Luiz et } \\
\text { al }\end{array}$ & $\begin{array}{l}\text { Investigar o uso de substâncias } \\
\text { estimulantes do sistema nervoso central } \\
\text { pelos estudantes de graduação em } \\
\text { Medicina da Universidade Federal do } \\
\text { Rio Grande, verificando as substâncias } \\
\text { mais utilizadas, os motivos de uso e o } \\
\text { perfil dos usuários }\end{array}$ \\
\hline & $\begin{array}{llr}\text { Metilfenidato e } & \text { aprimoramento } \\
\text { cognitivo } & \text { farmacológico: } \\
\text { representações } & \text { sociais de } \\
\text { universitários } & & \end{array}$ & $\begin{array}{l}\text { BARROS, Denise; } \\
\text { ORTEGA, Francisco }\end{array}$ & $\begin{array}{l}\text { Apresentar o resultado da investigação } \\
\text { das representações sociais de } 20 \\
\text { estudantes universitários sobre o uso de } \\
\text { metilfenidato para aprimorar o } \\
\text { desempenho cognitivo em pessoas } \\
\text { saudáveis }\end{array}$ \\
\hline
\end{tabular}

Fonte: Dados da pesquisa. 


\section{DISCUSSÃO}

Estudos que avaliaram o uso de metilfenidato em pelo menos uma vez durante a vida universitária identificou uma prevalência entre 8,3\% e 9\% (TETER et al., 2006, p. 1503). O estudo de Finger, Silva e Falavigna (2013, p. 285-289) avaliou a prevalência do uso de metilfenidato entre uma população de estudantes do $5^{\circ}$ e do $6^{\circ}$ ano de uma faculdade de medicina no sul do Brasil e encontrou que 34,2\% usavam metilfenidato, dos quais $23 \%$ o utilizavam sem razões médicas. Esse perfil é bem descrito em diversos estudos por todo o mundo. De acordo com essa revisão, um importante fator de risco para o uso não prescrito de MPH se dá pelo ambiente altamente competitivo de estudo, e ainda, com pessoas de poder aquisitivo desiguais.

O padrão das amostras encontradas nos estudos corresponde a estudantes do sexo feminino, etnia branca, com idade média de 25 anos, de estado civil solteiro e sem filhos, oriundos de cidades diferentes da faculdade, com alguma reprovação, matriculados em cursos da área de humanidades e de instituições privadas, com média de sono por dia de 6,5 horas, e avaliação da qualidade de saúde como regular ou ruim.

Em um estudo envolvendo 3.500 estudantes de uma universidade pública nos EUA, $79 \%$ daqueles que fizeram uso não prescrito começou a usá-lo durante a faculdade (TETER et al., 2003, p. 613). Adiciona-se a isso, o fato que ao comparar pessoas da mesma idade, a prescrição de psicoestimulantes entre estudantes universitários é mais prevalente. Achados sustentam a ideia de ser um estudante universitário, um fator de risco para o uso não terapêutico de metilfenidato (FINGER; SILVA; FALAVIGNA, 2013, p. 288). Esses grupos têm razões diferentes para o uso da droga; aqueles que começaram a usar metilfenidato durante a faculdade tem um propósito acadêmico, por outro lado, o grupo que começou a usar metilfenidato antes da faculdade o fez como um narcótico, para perder peso, ou mesmo para descobrir seu efeito.

A prevalência de consumo de estimulantes apresentou um padrão diferenciado quanto aos períodos do curso, sendo maior nos períodos finais e iniciais. Isso pode ser justificado pela fato de que nas séries iniciais há uma mudança na rotina dos estudantes ao ingressarem um curso superior, e nos períodos finais há uma maior busca pela qualificação profissional e inscrição em exames de seleção no mercado de trabalho, resultando no maior índice do consumo de psicoestimulantes (MORGAN et al., 2017, p. 106). 
Dentre as diversas motivações para usar MPH apresentadas nos estudos analisados, estão: ajudar nos estudos; melhorar a concentração, raciocínio e memória; experimentação; para ficar acordado; para ir às festas, para perder peso; ter mais energia; ter maior autoconfiança devido a pressão ambiental; pressão dos amigos. Um fato importante observado nos estudos é que o efeito benéfico sobre memória, raciocínio e concentração foi mais frequente entre aqueles que usaram estimulantes com essa finalidade, sugerindo que o motivo de uso tem influência direta no efeito relatado (CESAR et al., 2012, p. 185).

Entre os usuários pesquisados, foi considerável o número daqueles que fizeram o uso de MPH simultâneo com álcool, o que representa potencial risco à saúde devido as maiores chances de abuso e dependência do álcool e ainda apresentação de efeitos diversos do medicamento decorrentes da associação (SILVEIRA et al., 2014, p. 105).

Outras considerações importantes colhidas nos estudos são: é frequente a obtenção dos medicamentos gratuitamente de colegas; aqueles que não fazem uso de psicoestimulantes relatam sentir pressionados com o fato de colegas estarem fazendo aprimoramentos cognitivos para preparação aos testes de qualificação; há maior prevalência de uso de metilfenidato no grupo de alunos com médias acadêmicas baixas; o uso de MPH é mais frequente entre os alunos que residem em alojamentos e repúblicas.

Sobre os potenciais benefícios deste medicamento em indivíduos que não sofrem de Transtorno do Déficit de Atenção com Hiperatividade (TDAH), estudos existentes não mostram evidências consistentes de otimização sobre o SNC, apenas um artigo sugerindo efeito positivo na memória. Menor ainda é o número de estudos que analisam os possíveis efeitos colaterais em indivíduos saudáveis sem indicação, sendo descritos efeitos benignos, como taquicardia, alucinações visuais, vertigem, sonolência, cefaleia, falta de apetite, náuseas, ansiedade e distúrbios do sono (BRANT; CARVALHO, 2012, p. 627).

Os discursos dos universitários quanto ao uso do metilfenidato para melhorar o desempenho acadêmico pautaram-se sobre pontos importantes no contexto de saúde pública. A pressão social para melhorar o desempenho foi a principal motivação levantada, onde surgiu a percepção de que a cobrança social exigia um comportamento sobre-humano e uma postura competitiva, tanto com os outros como consigo mesmo, e essa prática no contexto do ambiente acadêmico é tida como desleal, uma vez que o fármaco melhoraria uma capacidade que a pessoa não tem naturalmente (BARROS; ORTEGA, 2011, p.353). 


\section{CONSIDERAÇÕES FINAIS}

Há um número insuficiente de estudos que tratam sobre o uso de psicoestimulantes entre estudantes saudáveis na literatura brasileira. Assim, é preciso avaliar os impactos do uso de estimulantes na prática clínica, a fim de minimizar possíveis efeitos adversos, avaliar a dose considerada segura e coibir o uso de determinadas substâncias.

As principais preocupações com a prática estão relacionadas ao sério risco de problema de saúde pública pela incorporação de outras drogas ao uso indevido de psicoestimulantes, e ainda, desta intensificar injustiças e desigualdades entre as pessoas em sociedades que já apresentam significativas diferenças sociais.

O conhecimento do perfil dos usuários pode ser de extrema importância na construção de políticas e estratégias focadas na prevenção do uso destas substâncias. O sistema público de saúde, bem como as universidades tem a responsabilidade de conduzir pesquisas e intervir no consumo de estimulantes entre acadêmicos. Assim, é importante realizar um diagnóstico das instituições educacionais para que se implementem medidas preventivas, visto que existe grande relação entre o uso de estimulantes e o consumo de álcool, cigarro, maconha e cocaína, o que representa grandes riscos à saúde do usuário.

\section{REFERÊNCIAS BIBLIOGRÁFICAS}

BARROS, D.; ORTEGA, F. Metilfenidato e aprimoramento cognitivo farmacológico: representações sociais de universitários. Saude soc., São Paulo, v.20, n.2, p.350-362, junho 2011.

BOTELHO, L. L. R.; CUNHA, C. C. A.; MACEDO, M. O método da revisão integrativa nos estudos organizacionais. Gestão e Sociedade, v. 5, n. 11, p. 121-136, abril 2011.

BRANT, L. C.; CARVALHO, T. R. F. Metilfenidato: medicamento gadget da contemporaneidade. Interface (Botucatu), Botucatu, v.16, n.42, p.623-636, setembro 2012.

BROOME, M. E. Integrative literature reviews for the development of concepts. In: RODGERS, B.L; CASTRO, A.A. Revisão sistemática e meta-análise. 2006.

CESAR, E. L. R. et al. Uso prescrito de cloridrato de metilfenidato e correlatos entre estudantes universitários brasileiros. Rev. psiquiatr. clín., São Paulo, v.39, n.6, p.183-188, outubro 2012. 
FINGER, G.; SILVA, E. R.; FALAVIGNA, A. Use of methylphenidate among medical students: a systematic review. Rev. Assoc. Med. Bras., São Paulo, v.59, n.3, p.285-289, junho 2013.

MORGAN, H. L. et al. Consumo de Estimulantes Cerebrais por Estudantes de Medicina de uma Universidade do Extremo Sul do Brasil: Prevalência, Motivação e Efeitos Percebidos. Rev. bras. educ. med., Rio de Janeiro, v.41, n.1, p.102-109, janeiro 2017.

SILVEIRA, R. R. et al. Patterns of non-medical use of methylphenidate among 5th and 6th year students in a medical school in southern Brazil. Trends Psychiatry Psychother, Porto Alegre, v.36, n.2, p.101-106, junho 2014.

TETER, C. et al. Illicit methylphenidate use in an undergraduate student sample: prevalence and risk factors. Pharmacotherapy, Boston, v. 23, n. 8, p. 609-617, fevereiro 2003.

TETER, C. et al. Illicit use of specific prescription stimulants among college students: prevalence, motives, and routes of administration. Pharmacotherapy, Boston, v. 26, n. 10, p. 1501-1510, outubro 2006. 